\title{
Novel sensor geometry for liquids serving in dispersion thermal flow meters.
}

\author{
Anastasios Badarlis $^{1,2}$, Vivek Kumar ${ }^{2}$, Axel Pfau ${ }^{2}$ \\ Anestis Kalfas \\ ${ }^{1}$ Aristotle University of Thessaloniki \\ ${ }^{2}$ Endress+Hauser Flowtec AG
}

\section{Nomenclature}

\begin{tabular}{|c|c|}
\hline$\dot{Q}$ & Generated heat on heater surface [W] \\
\hline$T_{\text {heater }}$ & Temperature of the heater $[\mathrm{K}]$ \\
\hline & Process temperature $[\mathrm{K}]$ \\
\hline$P C$ & Power coefficient $\left[\mathrm{W} \mathrm{K}^{-1}\right]$ \\
\hline$R_{\text {solid }}$ & Thermal resistance in solid domain $\left[\mathrm{K} \mathrm{W}^{-1}\right]$ \\
\hline$R_{\text {fluid }}$ & Thermal resistance in fluid domain $\left[\mathrm{K} \mathrm{W}^{-1}\right]$ \\
\hline$h_{\text {conv }}$ & Heat transfer coefficient $\left[\mathrm{W} \mathrm{m}^{-2} \mathrm{~K}^{-1}\right]$ \\
\hline$L_{c h a r}$ & Characteristic length [m] \\
\hline$K_{\text {solid }}$ & Solid domain thermal conductivity $\left[\mathrm{W} \mathrm{m}{ }^{-1} \mathrm{~K}^{-1}\right.$ \\
\hline$T_{\text {ext }}$ & $\begin{array}{l}\text { Temperature of the additional concentric } \\
\text { temperature meter (external ring) }[\mathrm{K}]\end{array}$ \\
\hline$T_{\text {interface }}$ & Solid-fluid domain interface temperature $[\mathrm{K}]$ \\
\hline$U_{\text {inlet,profile }}$ & Inlet flow velocity $[\mathrm{m} / \mathrm{s}]$ \\
\hline$U_{\text {mean }}$ & Mean flow velocity $[\mathrm{m} / \mathrm{s}]$ \\
\hline$r$ & Distance measured from the pipe center [m] \\
\hline$R_{\text {pipe }}$ & Pipe radius $[\mathrm{m}]$ \\
\hline$k$ & Turbulent kinematic energy $\left[\mathrm{m}^{2} \mathrm{~s}^{-2}\right]$ \\
\hline$\omega$ & Turbulent frequency $\left[\mathrm{s}^{-1}\right]$ \\
\hline$\rho$ & Medium density $\left[\mathrm{Kg} \mathrm{m}^{-3}\right]$ \\
\hline$\nu t$ & Kinematic eddy viscosity $\left[\mathrm{m}^{2} \mathrm{~s}^{-1}\right]$ \\
\hline$\mu_{t}$ & Dynamic eddy viscosity $\left[\mathrm{Kg} \mathrm{m} \mathrm{s}^{-1}\right]$ \\
\hline$\dot{q}$ & Heat flux $\left[\mathrm{W} \mathrm{\textrm {m } ^ { - 2 } ]}\right.$ \\
\hline$T_{i}$ & Local temperature in i position $[\mathrm{K}]$ \\
\hline$\dot{m}$ & Mass flux $\left[\mathrm{Kg} \mathrm{m}^{-2} \mathrm{~s}^{-1}\right]$ \\
\hline
\end{tabular}

\section{Dimensionless}

$\begin{array}{ll}\mathrm{Re} & \text { Reynolds number } \\ \mathrm{Nu} & \text { Nusselt number } \\ \mathrm{Bi} & \text { Biot number } \\ \mathrm{Pr} & \text { Prantl number }\end{array}$

\section{Introduction}

\subsection{General}

In many industrial plants the flow measurement is one of the most crucial information of the application. A lot of different flow meters based on different principals have been developed, some of them are more favorable in some applications and flow mediums than the others. Thermal flow meter is one common type of flow-meter. Two variations of thermal flow meters are mainly used; the dispersion and the capillary-tube thermal flow meter. Of course, there are and other thermal flow meters measurement principals, but they are not common in industrial application. One example is the time-of-flight which is described in Ashauer et al., 1999 [1]. This type of flow-meter measures the flow velocity.

Both dispersion thermal flow meters and capillarytube thermal flow meter measures mass flow rate, but the direct measurement quantity is the heat flow rate. Heat dissipates from a heated surface to the flowing fluid. In the case of the thermal dispersion type, the heat is transferred, from the heater to the boundary layer of the fluid flowing over the heated surface. Thermal dispersion flow meters are commonly used for general industrial flow applications in pipes and ducts.

Capillary type is primarily used for smaller flows of clean gases or liquids in tubes, Viswanathan et al., [2] and Amador et al., [3]. Capillary flow meter is not the subject of this investigation.

The dispersion type thermal flow meter comprises two Pt-100 elements which one of them has heater and temperature measurement functionalities simultaneously, while the second one has only temperature measurement functionalities and measures the temperature of the process. Both of the Pt-elements are soldered on the internal flat face of the cap [Fig. 1 a]. The cap has $18 \mathrm{~mm}$ of shaft diameter. The cap is welded with a probe. The measurement can be conducted either under constant power mode (the supplied power is constant) or under constant temperature difference (controlling the provided power on the heater).

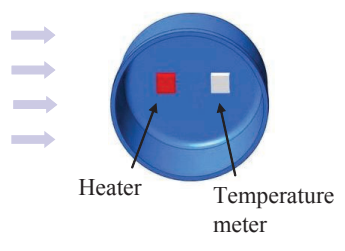

(a)

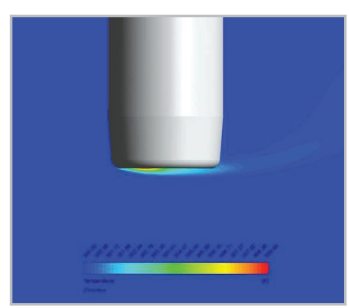

(b)

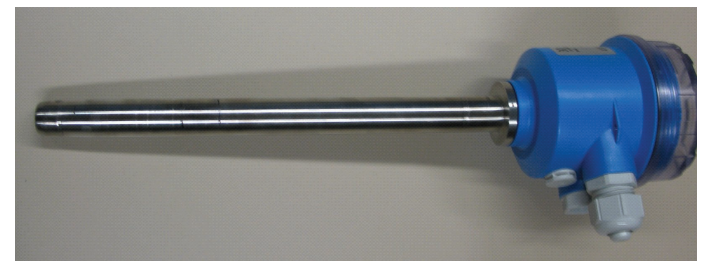

(c)

Fig. 1. Dispersion thermal flow meter geometry in liquids applications. Pt-elements in the internal side of the cap (a). Probe of the flow meter in the flow and temperature contours (b). Insertion dispersion thermal flow meter for liquids (c).

The objective of this work was the numerical investigation of the dispersion thermal flow meter [Fig. 1]. This investigation helped to understand better the way of working, the heat flow and the temperature 
distribution in the sensor. Furthermore, the flow formation on the region close to Pt-elements position was an important aspect of this numerical investigation.

The second part of this numerical investigation regards ways of improving the sensitivity of the characteristic curve, this can be done following two ways. Firstly, by optimizing the position of the heater with criterion the position that gives the characteristic curve with the less curvature. Secondly, by a new heater design (proposed model) that is expected to improve the gradient of the characteristic curve in high flow rates, making the sensor more sensitive in flow rate changes.

\subsection{Theory and Sensor}

The measurement quantity, in the current dispersion thermal flow meters, is the power coefficient, which consist of the ratio of the generated energy to the temperature difference between the heater and the process [Eq. 1].

$$
\begin{aligned}
& P C=\frac{\dot{Q}}{T_{\text {heater }}-T_{\infty}} \\
& P C=\frac{1}{R_{\text {solid }}+R_{\text {fluid }}}
\end{aligned}
$$

Characteristic curve of the dispersion thermal flow meters (Fig. 2) have exponential form. From Fig. 2 it implies that the most favorable curve is curve 1 . This statement can be based on two arguments. The first is the lower curvature of curve 1 comparing to curve 2. The second argument, which makes curve 1 more favorable, is the fact that curve 1 has higher gradient in high flow rates, this makes the flow meter more sensitive.

$$
\begin{aligned}
& P C=\frac{h_{\text {conv }} A}{1+B i} \\
& B i=\frac{h_{\text {conv }} L_{\text {char }}}{K_{\text {solid }}}
\end{aligned}
$$

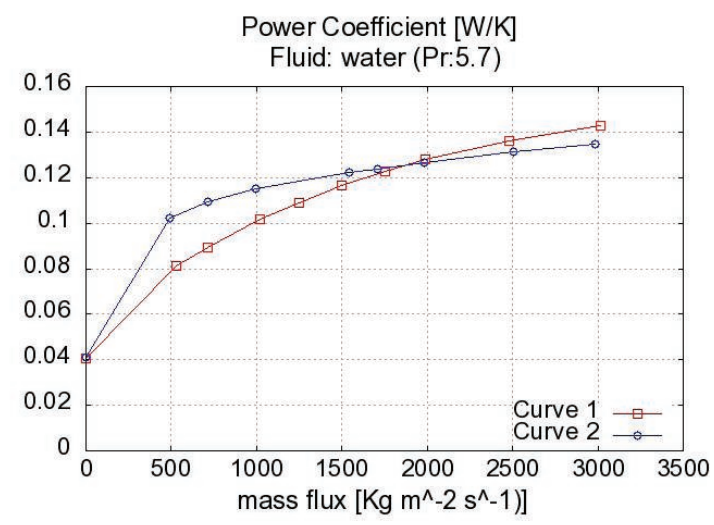

Fig. 2. Characteristic curves of a thermal flow meter in water. Curve 1 has more sensitive characteristic, its curvature is lower than the curvature of curve 2 and in high flow rates it has higher gradient than curve 2 .

Analyzing the physics behind the dispersion thermal flow meters, the measurement quantity is equal to the reverse of the overall thermal resistance. The overall thermal resistance consists of the fluid and solid thermal resistance [Eq. 2]. In gas applications fluid thermal resistance has the dominant influence in the quantity of power coefficient. While in case of liquids the solid domain thermal resistance influence can not be considered negligible as in gases, so changes of the thermal resistance based on flow rate changes, can not be so easy detectable, this makes the flow meter less sensitive. Bi number (Eq. 4) increases as heat transfer coefficient increases, but this makes the power coefficient less sensitive. As it is presented in Eq. 3, which equation describes physics, Bi number is appeared in the denominator of the equation. The ideal measurement would be in the case of Bi number equal to zero, then the power coefficient becomes proportional to heat transfer coefficient, which is depended only on the fluid flow and fluid properties. One additional disadvantage of the low sensitivity of the curve in high flows can be considered the difficulty of finding the adequate mathematical model, which can correlate the calibration data.

The main problem in liquid applications comes in high flows (higher than $2000 \mathrm{Kg} /\left(\mathrm{m}^{2} \mathrm{~s}\right.$ ) in water), where the signal of the measurement quantity (power coefficient) becomes weak and the signal to noise ratio approaches the one. The reason of low sensitivity in high flow can be explained by the Eq. 3 . $\mathrm{Bi}$ in high flows number can reach the values more than 3.

The proposed design (Fig. 3) has the heater in round shape in the middle of the substrate and an additional concentric temperature meter (external ring), the temperature meter of the process is not visible on this figure. The power coefficient, in this case is defined as the ratio of the generated heat on the heater divided by the temperature difference between the additional temperature meter (Eq. 5) and the process temperature. Following this approach the influence of the solid domain (sensor) is expected to be reduced and the sensitivity of the characteristic curve in high mass flow rates to be improved. This can happen if the external temperature ring represents the temperature of the fluid-solid interface.

Additional Temperature meter

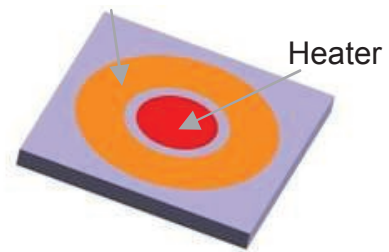

(a)

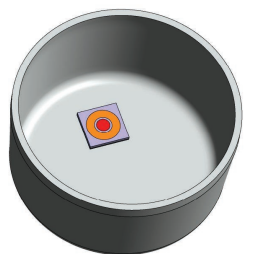

(b)
Fig. 3. Proposed heater design, with an additional temperature meter (orange ring) (a). Cap, substrate soldered on the internal flat surface of the cap (b).

$P C=\frac{\dot{Q}}{T_{\text {ext }}-T_{\infty}}$

Previous publications, which can be related with this type of geometries, focused on cylinders with free end. Experimental investigation has been conducted by Ghisalberti and Kondjoyan 2002, 1999 [4], [8], authors conducted visualization of the heat transfer coefficient around cylinder with free end, as relation of flow velocity and turbulence, while they tried to explain flow patterns. Park and Lee 2000 [10] investigated the free end influence on the flow formation and the variations with the infinite cylinder problem, like how this influences the formation of the 
flow and the heat transfer. Nakamura et al., 2004 [11], was focused on the fluctuating heat transfer in the unsteady regions on the surface of a circular cylinder. One of the few studies which investigated circular cylinders heat transfer in liquids, conducted by Sanitjai and Goldstein 2004 [9], the authors measured the local heat transfer coefficient around the cylinder and made proposal for correlating equations.

Numerical investigations for finite cylinder have been conducted by Afgan et al., 2007 [12] in this paper finite cylinder problem was solved with LES model and compared with experimental results. Another numerical investigation has been conducted by Pattenden et al. [14], they solved the problem of finite cylinder, using LES and DES models for Re 200000 and finally compared the results to experimental data. According to they results, both LES and DES can not predict precisely the formation of the flow on the free end of the cylinder.

\section{Methods}

\subsection{Numerical model}

Finite volume method was employed for the current investigation with conjugate heat transfer. Conjugate heat transfer allows the coupled heat transfer solution between solid and fluid, and thereby more accurate heat transfer predictions. The energy equation for solids is a degenerate form of the energy equation for fluids (obtained by setting the velocity to zero). Flow meter was placed in pipe of diameter $0.13 \mathrm{~m}$ and $0.5 \mathrm{~m}$ long, insertion depth was $50 \%$ and the distance from the inlet was $20 \%$. As fluid, water was selected ( $\mathrm{Pr}$ 5.7) under pressure of 1 atm, fluid is considered incompressible. Flow velocity range started from $\mathrm{Re}$ 300 up to $\operatorname{Re} 75000$. The model solved for 7 different Re numbers. ANSYS CFX commercial software was used for the setup of the problem, the solution and the post processing of the results.

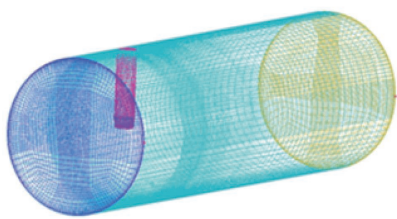

(a)

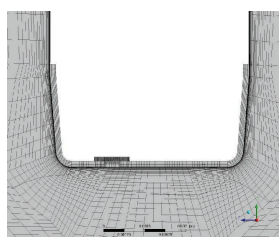

(b)
Fig. 4: Finite volume model (a). Section view of the grid around the sensor (b).

Finite volume model Fig. 4 was discretized with ICEM CFD commercial software and was divided in two domains the solid and the fluid. Solid domain consists of 63169 volume elements and the fluid domain consists of 462180 hexa-elements. The grid of the fluid domain around the cap, which is the region of high heat transfer exchange, was very fine and discretized according to $\mathrm{Y}^{+}=1$ for $\operatorname{Re} 75000$.

According to boundary conditions of the model, the inlet flow profile was set as fully developed turbulent Eq. 6 and turbulence intensity 5\%. The pipe and probe walls were selected as no slip smooth wall. The outlet was defined so that the outlet average static relative pressure was equal to zero.

SST turbulence model combines the advantages of the K-epsilon and K-omega models, blending between k-omega model in the near surface regions and k-epsilon in the outer region.

$$
\begin{array}{ll}
U_{\text {inlet, profile }}=1.18 \cdot U_{\text {mean }} \cdot\left(1-\frac{r}{R_{\text {pipe }}}\right)^{\frac{1}{7}} & \text { Eq. } 6 \\
\frac{\partial \rho k}{\partial t}+\nabla \cdot(\rho \overrightarrow{U k})=\nabla \cdot\left[\left(\mu+\frac{\mu_{t}}{\sigma_{k 3}}\right) \nabla k\right] & \text { Eq. } 7 \\
+P_{k}+P_{k b}-\beta^{\prime} \rho k \omega & \\
\frac{\partial \rho \omega}{\partial t}+\nabla \cdot(\rho \vec{U} \omega)=\nabla \cdot\left[\left(\mu+\frac{\mu_{t}}{\sigma_{\omega 3}}\right) \nabla \omega\right]+ & \text { Eq. } 8 \\
\left(1-F_{1}\right) 2 \rho \frac{1}{\sigma_{\omega 2} \omega} \nabla k \nabla \omega+\alpha_{3} \frac{\omega}{k} P_{k}+P_{\omega b}-\beta_{3} \rho \omega^{2} & \\
\nu_{t}=\frac{\alpha_{1} k}{\max \left(\alpha_{1} \omega, S F_{2}\right)}, \quad \nu_{t}=\frac{\mu_{t}}{\rho} & \text { Eq. } 9
\end{array}
$$

$F_{1}$ and $F_{2}$ are blending functions, which restricts the limiter to the wall boundary layer. The eddy-viscosity is calculated from Eq. 9. The other parameter of the Eq. 7, Eq. 8 and Eq. 9, are constant coefficient or are calculated by additional number of functions as presented in the manual of commercial CFD software ANSYS CFX [7]

The wall treatment of the near-wall region is critical for the accuracy of the results. CFX offers the functionality of wall treatment as "automatic". This works favorable with k-omega turbulence model which is used by SST model in the close to surface regions. "Automatic" wall treatment has the advantage of switching between wall functions in coarse mesh regions to low-Re method in refined mesh regions. Low-Re method is much more accurate, but it has high computation cost. For the higher accuracy of the results, high resolution advection scheme was employed.

Steady state solution was employed as the most adequate, because the working mode of the sensor can be described as steady state. Moreover, it is much faster than the unsteady solution. Furthermore, the signal of the sensor is not influenced by the shedding vortices on the flat face region, because the time constant of the solid domain is much higher than the shedding vortices period, so the solid domain works as filter of the high frequency shedding vortices. In addition, this validates the use a time averaging turbulence model like SST.

\subsection{Optimization Procedure for Heater Position}

For the calculation of the optimal position of the heater in the internal flat face of the cap the following procedure was adopted. The model during this simulation did not contain the heater, the substrate of the heater and the solder material, it consist of the fluid domain, the cap and the probe. Firstly, constant heat flux on the internal flat face of the cap was provided, and then the model was solved for different flow velocities. For the whole flat face, a local quantity, identical to power coefficient was calculated Eq. 10. This calculation provided, for each point on the flat face discrete data PC/A against flow, which in reality is a quantity similar to the characteristic curve of each point. In second step, the gradients (Eq. 11) between the discrete measurement points, of each characteristic curve (characteristic quantity in Eq. 10) was calculated. In the final step, the variance of the gradients (Eq. 12) for each characteristic curve was derived. The criterion of the optimization is based on gradient distribution (variance) of the characteristic curve. The minimum of this quantity defines the 
position in the flat face with the less variance of the gradient. In other words, the procedure tries to identify the position with the less absolute curvature of the characteristic curve (Eq. 10). The position on which, if the heater is place, it will provide the less bended characteristic curve.

$\frac{P C}{A}=\frac{\dot{q}}{T_{i}-T_{\infty}}$

$\operatorname{grad}\left(\frac{P C}{A}\right)_{i+1}=\frac{(P C / A)_{i+1}-(P C / A)_{i}}{\dot{m}_{i+1}-\dot{m}_{i}}$

$\operatorname{Var}(\operatorname{grad})=\frac{1}{N-1} \sum_{2}^{N}\left(\operatorname{grad}_{j}(P C / A)-\overline{\operatorname{grad}(P C / A)}\right)^{2}$

\section{Results and Discussion}

\subsection{Flow Field}

Analyzing the results, interesting aspects of the flow formation around the cap and particularly on the flat surface of the sensor has been revealed.

Projecting the streamlines in a plane, which is the symmetric plane of the fluid domain, the formation of a recirculation on the flat face of the sensor was observed (Fig. 5). The flow separates on the front edge of the cap, while further downstream reattaches on the flat face. The reattachment position (Fig. 6) varies and moves upstream as the $R e$ number increases. Another observation on Fig. 5, is the height of the recirculation which decreases as Re increases. In the near-wake, it is also noticeable the formation of a secondary flow, which generates a vortex with its axis vertical to probe axis. This vortex is responsible for the elongation of the eddy formation region $L_{f}$ and Strouhal number variation along the span of the cylinder, as Zdravkovich [5] presents in his book collecting experimental data from different researchers.

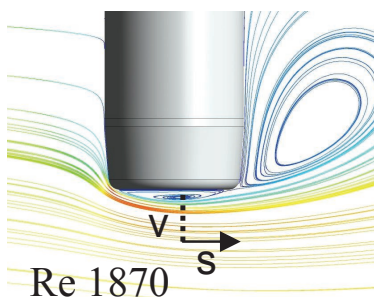

(a)

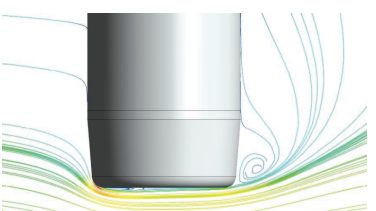

$\operatorname{Re} 74900$

(c)

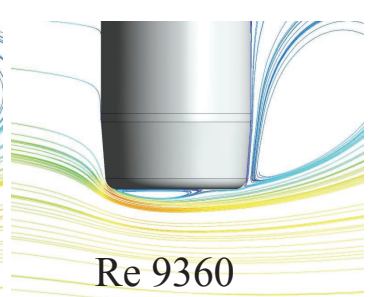

(b)

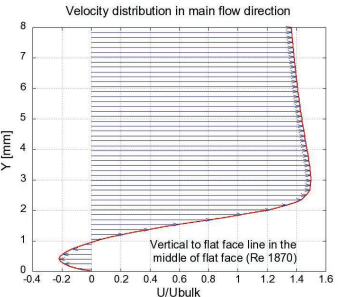

(d)
Fig. 5: Streamlines formation on a plate for different Re numbers (a), (b) and (c). Normalized velocity in flow direction component, through the line $v$ for $\mathrm{Re}$ 1870 (d).

The formed recirculation on the flat face of the cap is comprised by a secondary flow, which is generated by the pressure difference between the free stream and the flat face (free end).

Flow formation on the free end is 3-D and anisotropic. In Fig. 7, the owl-eyes vortices are formed in the separation area and they have their starting point adapted on the flat face. Vortices are elongated in direction normal to the main flow and formulate the recirculation.

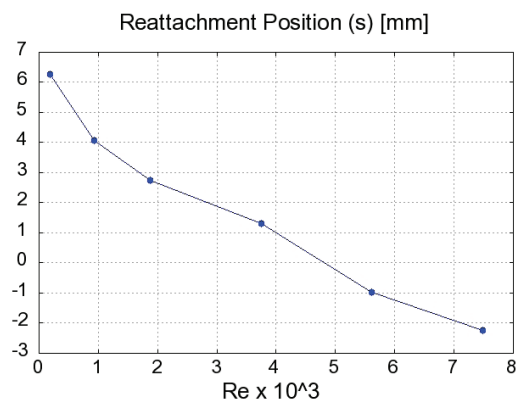

Fig. 6: Reattachment position of the recirculation on the flat face. The s distance has reference the middle of the flat face (Fig. 5 (a)). Recirculation is moving upstream.

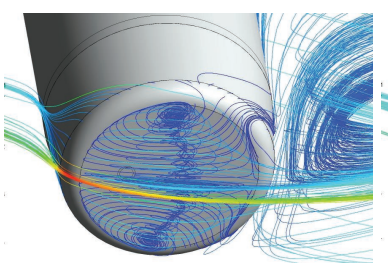

Re 1870

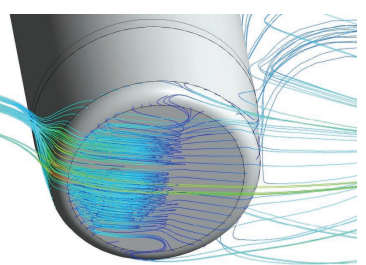

Re 18700
Fig. 7: Owl-eyes vortices.

\subsection{Heat Transfer Distribution}

Formation of the flow on the free end influences the local heat transfer. For the calculation of the heat transfer, constant heat flux on the outer surface of the cap, was provided. The results of this calculation are presented on Fig. 8.

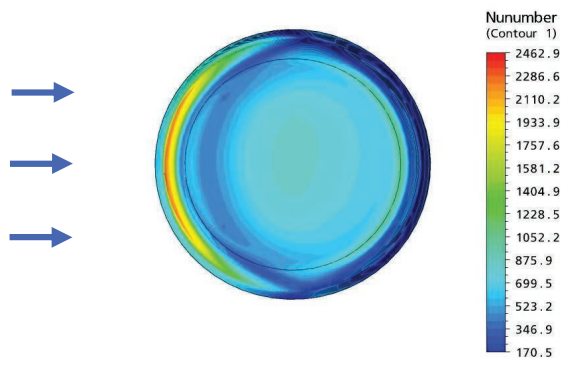

Fig. 8: Nu number on the cap for $\operatorname{Re} 37420$.

From Fig. 8 implies that the heat transfer on the upstream side of the flat face, where the recirculation is formed, has significantly lower heat transfer coefficient than the downstream part, where there is formation of a new boundary layer, as it can be observed on Fig. 5 and Fig. 7. The new boundary layer means that the heat transfer coefficient has higher values in this region and the thermal resistance in fluid boundary layer is lower. The highest heat transfer is observed on the stagnation front area in the area of the fillet.

\subsection{Optimal position of the Heater}

One of the critical decisions on the sensor design is 
related to the position of the heater in the internal flat face of the cap. The calculation of the optimal position has been presented on methods chapter (2.2), the criterion was the minimum of the characteristic curve gradient variance. Or in other words, the characteristic curve with the less curvature, the position which corresponds in this curve is the most favorable.

Calculating the variance of the characteristic curve gradients Fig. 9 is derived. Taking into consideration that the best performance of the characteristic curve has the area with the less variance, it implies that the half upstream area of the cap is the best for the positioning of the heater.

The variance in the half downstream part follows an increasing trend from the middle of the face in direction downstream and reaches it maximum values on the downstream edges.

With purpose to understand better the variation of the characteristic curves in the different position of the cap, Fig. 10 has been introduced. This presents the characteristic curve of three different points, it should be mentioned that for the calculation of these curves the thermal resistance of the solder and the substrate of the heater has been added artificially, and was considered as constant. The area of heat transfer, on the cap - fluid interface, was considered 0.000013 $\mathrm{m}^{2}$. In that way, we could calculate the total power coefficient from PC/A by multiplying it with the considering area, this provides the $\mathrm{PC}_{\text {cap }}$, while in continue the thermal resistance of the solder and heater substrate was added (Eq. 13).

$$
P C_{\text {total }}=\frac{1}{\frac{1}{P C_{\text {cap }}}+R_{\text {solder, substr }}}
$$

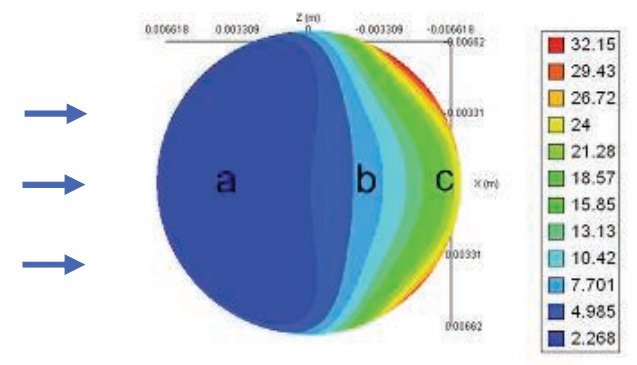

Fig. 9: Local variance of the characteristic curve gradients. The half upstream area provided characteristic curves which have less curvature than the downstream.

Normalized PC with c position maximum, Pr:5.7

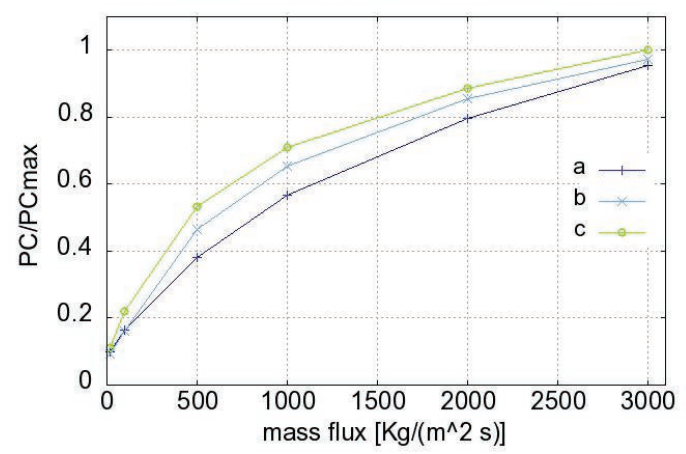

Fig. 10: Normalized power coefficient, the maximum corresponds to the maximum on $\mathrm{c}$ position.
In Fig. 10 the calculated power coefficient in these three points is normalized with the maximum $P C$ of "c" position. It is inferred that the heater in position "a" has characteristic curve with the less curvature while the gradient in high flow velocities is higher in "a" region. One additional observation, on Fig. 10 is related to the values of PC for each curve, on "a" region the $\mathrm{PC}$ is lower than the other two positions, this is reasonable, because in the region "a" the heat transfer coefficient is lower as it is presented in Fig. 8.

\subsection{Proposed Design Investigation}

Placing the sensor in the optimal position, which is derived from the previous investigation, the proposed design was investigated under coupled CFD heat transfer solution. On Fig. 11 the isothermals, which cross the outer surface, cross also the external temperature meter ring on the substrate, from this fact, it is inferred that the temperature of the solid-fluid interface (external side of the cap) can be estimated.

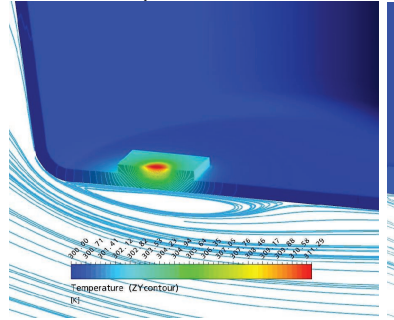

(a)

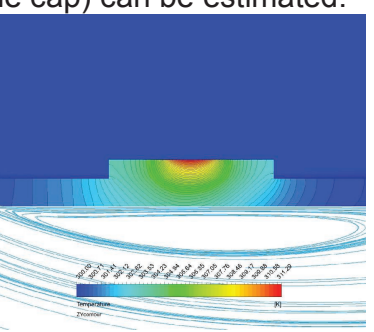

(b)
Fig. 11: Temperature distribution in a section-view of the sensor for $\operatorname{Re} 18700$ and $\operatorname{Pr} 5.7$ (water). The same isothermals cross the solid-fluid interface and the external temperature ring. 3-D view (a). 2-D view in the heater area (b). (Red: $310 \mathrm{~K}$, blue: $300 \mathrm{~K}$ )

The averaged temperatures on the surfaces of heater, additional temperature meter (external ring) and solid-fluid interface are presented on Fig. 12. The temperature of the heater is maintained constant through the whole measurement range (310K), which means that the sensor is in constant temperature difference mode, the process temperature was defined to $300 \mathrm{~K}$. The second observation is related with the temperature on the external ring and the solid-fluid interface, the two curves follows similar forms, as the flow increases, but they do not lay on each other. A further optimization of the sensor design can be done so that the two curves can come closer or become identical.

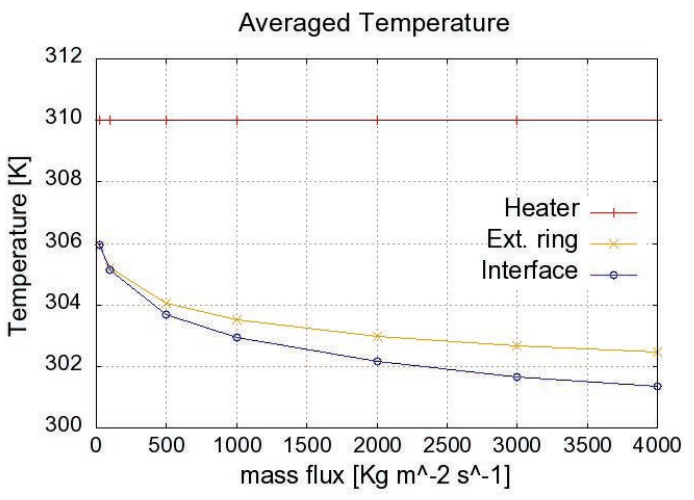

Fig. 12: Averaged temperature on different surfaces of the sensor. 
Comparing the three cases on Fig. 13, which describes the normalized power coefficient, the trend of the improvement with the new proposed design is distinguishable. In the optimal case, which is a theoretical case, power coefficient is calculated taking into account the temperature of the solid-fluid heated interface, as it is defined by Eq. 14. In optimal case there is no influence of the solid domain in the measurement quantity. In the standard approach, the temperature of the heater is used for the calculation of the power coefficient (Eq. 1). This means that the thermal resistance of the solid influences the measurement quantity. In the third case, which is the proposed one, the measurement quantity is calculated from the Eq. 5, the influence of the solid domain in this case is reduced and the gradient of the curve in high flow velocities is increased and approaches the gradient of the optimal case. The improvement of the characteristic curve gradient $[P C=f(\rho u)]$ in mass flux 3000 to $4000\left[\mathrm{Kg} \mathrm{m}^{-2} \mathrm{~s}^{-1}\right]$ is calculated in $41.8 \%$.

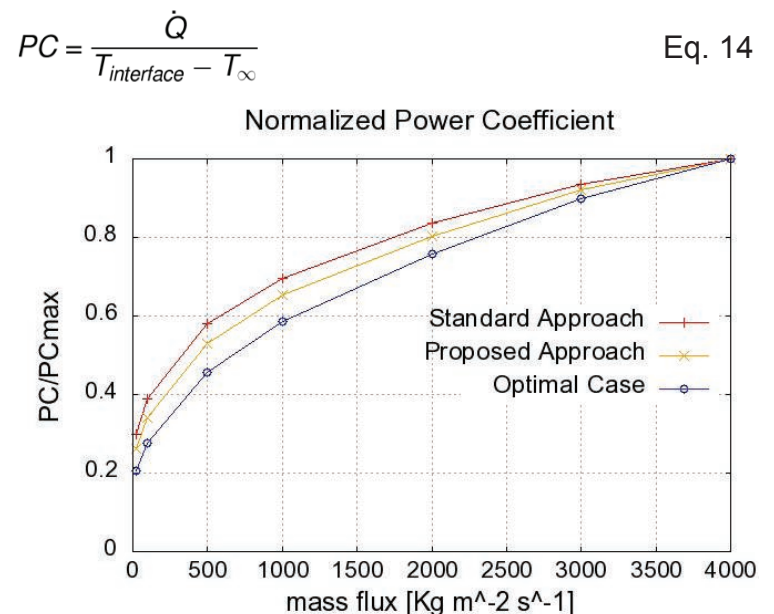

Fig. 13: Normalized power coefficient for the standard, the new proposed approach and the optimal.

\section{Conclusions}

From the current numerical investigation of the thermal flow meter in liquids, the following conclusions can be derived:

- The proposed design, as it was expected, has the trend to improve the sensitivity in high flow velocities. The gradient of the characteristic curve was increased by $41.8 \%$.

- The high sensitivity in high flow rates can increase the functional flow velocities range of the flowmeter.

- On the flat face, as and many other researchers has observed in free end cylinders, is formed a recirculation that consist of two vortices, these vortices have the characteristic name of owl-eyes.

- The recirculation on the flat face of the cap is critical for the performance of the flow meter, because it generates an area of low heat transfer coefficient.

- The vortex on the rear part of the cylinder in the near-wake region is formed and elongates the eddy formation region.

- The half upstream internal part of the cap, consist the area in which the characteristic curve has lower curvature and higher gradient in high flow rates, this makes the half upstream part the most favorable position for the heater.

\section{References:}

[1] Ashauer, M., Glosch, H, Hedrich, F., Hey N., Sandmaier H., Lang W., 1999. Thermal flow sensor for liquids and gases based on combinations of two principles. Sensors and Actuators, 73, 1999, 7-13.

[2] Viswanathan, M., Kandaswamy, A., Sreekala, S.K., Sajna, K.V., 2002. Development, modeling and certain investigations on thermal mass flow meters. Flow Measurement and Instrumentation 12, 353-360.

[3] Amador, M., A., Ferreira, V., 2010. Gas massflow meters: Principles and applications. Flow Measurement and Instrumentation 21, 143-149.

[4] Ghisalberti, I., Konjoyan A., 2002. Complete map out of the heat transfer coefficient at the surface of two circular cylinders $H / D=3.0$ and 0.3 subjected to a cross-flow of air. Int. J. of Heat and Mass 45, pp. 2597-2609.

[5] Zdravkovich, M., 1997. Flow Around Circular Cylinders. Oxford University Press.

[6] Davidson, L., 2003. An Introduction to Turbulence Models. Publication 97/2, CHALMERS, http://www.tfd.chalmers.se/ ₹lada.

[7] ANSYS CFX solver Theory guide. http://www.kxcad.net/ansys/ANSYS_CFX/help/h elp/Theory/.

[8] Ghisalberti, I., Konjoyan A., 1999. Convective heat transfer coefficients between air flow and a short cylinder. Effect of air velocity and turbulence. Effect of body shape, dimensions and position in the flow. Int. J. of Food Engineering 42, pp. 33-44.

[9] Sanitjai, S., Goldstein, R.J., 2004. Forced convection heat transfer from a circular cylinder in crossflow to air and liquids. Int. J. of Heat and Mass Transfer 47, 4795-4805.

[10] Park, C.W., Lee, S.J., 2000. Free end effects on the near wake flow structure behind a finite circular cylinder. J. of Wind Engineering and Industrial Aerodynamics 88, 231-246.

[11] Nakamura, H., Igarashi, T., 2004. Unsteady heat transfer from a circular cylinder for Reynolds numbers from 3000 to 15000 . Int. J. of Heat and Fluid Flow 25, 741-748.

[12] Afgan, I., Moulinec, C., Prosser, R., Laurence, D., 2007. Large eddy simulation of turbulent flow for wall mounted cantilever cylinders of aspect ratio 6 and 10. International Journal of Heat and Fluid Flow 28, 561-574.

[13] Incropera, F.P, Dewitt, D. P., 1996. Fundamental of Heat and Mass Transfer. 4th Edition, John Willey \& sons.

[14] Pattenden, R.J., Bressloff, N.W., Turnock, S. R., and Zhang, X., 2007. Unsteady simulations of the flow around a short surface-mounted cylinder. Int. J. Numer. Meth. Fluids, 53, 895914.

\section{Acknowledgement:}

The authors would like to thank Endress+Hauser Flowtec AG for the possibility to publish this work. 